\title{
A NONLINEAR STURM-LIOUVILLE PROBLEM ${ }^{1}$
}

\author{
BY JAY H. WOLKOWISKY
}

Communicated by E. Isaacson, May 16, 1967

We consider the following nonlinear boundary value problems on the interval $\alpha \leqq x \leqq \beta$ :

$$
\begin{gathered}
{\left[p(x) y^{\prime}\right]^{\prime}+q(x) y+\lambda y\left[a(x) \pm h\left(x, y, y^{\prime}\right)\right]=0,} \\
y(\alpha)+\gamma_{1} y^{\prime}(\alpha)=0, \quad y(\beta)+\gamma_{2} y^{\prime}(\beta)=0 .
\end{gathered}
$$

Here $\lambda$ is a real constant. We assume that $p(x)>0, a(x)>0, p^{\prime}(x)$, and $q(x)$ are continuous in $\alpha \leqq x \leqq \beta$. In addition in the region

$$
D=\{(x, y, z) \mid \alpha \leqq x \leqq \beta,-\infty<y<\infty,-\infty<z<\infty\},
$$

we require $h$ to satisfy the following conditions:

$$
h(x, y, z) \text { is defined and continuous, }
$$

$$
\begin{aligned}
& h(x, y, z) \geqq 0, \\
& h(x, 0,0)=0,
\end{aligned}
$$

$$
\lim _{c \rightarrow+\infty} h(x, c \xi, c \eta)=\infty \text { uniformly for } \alpha \leqq x \leqq \beta
$$

and for all $\xi \neq 0, \eta \neq 0$.

We also assume that $\gamma_{1}, \gamma_{2}$, and $q(x)$ are such that the eigenvalues, $\lambda_{n}$, of the "linearized" problem,

$$
\left[p(x) \hat{y}_{n}^{\prime}\right]^{\prime}+q(x) \hat{y}_{n}+\lambda_{n} a(x) \hat{y}_{n}=0
$$

with boundary conditions (2), satisfy

$$
0<\lambda_{1}<\lambda_{2}<\cdots<\lambda_{k}<\cdots,
$$

with $\hat{y}_{k}$ having $k-1$ zeros in the open interval $(\alpha, \beta)$.

Previous studies of special cases of equations $\left(1^{+}\right)$and $\left(1^{-}\right)$with boundary conditions (2) have been done by Ljusternik [4], Nehari [5] and Pimbley [6], [7] among others. Similar nonlinear eigenvalue problems for partial differential equations have been treated by Berger [1], Browder [2], and Levinson [3].

We treat the question of the existence and multiplicity of solutions of equations $\left(1^{+}\right)$and $\left(1^{-}\right)$with boundary conditions (2). There are

1 This paper represents results obtained at the Courant Institute of Mathematical Sciences, New York University, under the sponsorship of the National Science Foundation, Grant NSF-GP-6724. 
two different kinds of existence behavior, depending on whether $\left(1^{+}\right)$ or $\left(1^{-}\right)$is considered. The following two theorems indicate these differences. These results also give an answer to the question of whether the nontrivial solutions (eigenfunctions) given by the linearized theory for particular values of $\lambda$, continue to exist as nontrivial solutions for the nonlinear equation at other values of $\lambda$.

ThEOREM 1. For equation $\left(1^{+}\right)$with boundary conditions (2) and for each $\lambda \in\left(0, \lambda_{n}\right)$ there exist a denumerable infinity of pairs of real nontrivial solutions $\left(y_{k}, \bar{y}_{k}\right)$ for each integer $k \geqq n$. Moreover, each element of the kth pair has $k-1$ zeros in the open interval $(\alpha, \beta)$. In addition for $\lambda \leqq 0$ there exist no nontrivial solutions. Also for the special case in which $h(x, y, z)=h(x,-y,-z)$ on $D$, then $y_{k}=-\bar{y}_{k}$.

THEOREM 2. For equation (1-) with boundary conditions (2) and for each $\lambda \in\left(\lambda_{n}, \infty\right)$ there exist $n$ pairs of real nontrivial solutions $\left(y_{k}, \bar{y}_{k}\right)$, $k=1,2, \cdots, n$. Also for each $\lambda \in(-\infty, 0)$ there exist a denumerable infinity of pairs of real nontrivial solutions $\left(Y_{k}, \bar{Y}_{k}\right), k=1,2, \cdots$, which do not bifurcate from the trivial solution $y \equiv 0$. Moreover each element of the kth pair, in each of the above cases has $k-1$ zeros in the open interval $(\alpha, \beta)$. In addition for $0 \leqq \lambda<\lambda_{1}$ there exist no nontrivial solutions. Also for the special case in which $h(x, y, z)=h(x,-y,-z)$ on $D, y_{k}=-\bar{y}_{k}$ and $Y_{k}=-\bar{Y}_{k}$.

The proof of these theorems is based upon a method used by the author in [8]. In that case the problem concerned the existence of buckled states of a circular plate and a theorem analogous to Theorem 2 was proved. The method involves certain nonlinear operators. The properties of these operators depend very strongly on the theory of the linear Sturm-Liouville problem. These operators are shown to have fixed points by using the Schauder fixed point theorem. Then it is shown that the existence of these fixed points implies the existence of the solutions referred to in Theorems 1 and 2 .

Further results. 1. Condition (6) does not have to hold for all $\xi \neq 0, \eta \neq 0$ in special cases. For example, if $h(x, y, z)=(z-k y)^{2}, k$ constant, then for all $\xi \neq 0, \eta \neq 0$ such that $\eta=k \xi$ condition (6) is not satisfied. Nevertheless, it can be shown that the statements of the above theorems are still true.

2. The restriction (8) that all the linearized eigenvalues be positive can be dropped and similar results hold. However the special cases $\lambda<0, \lambda=0$, and $\lambda>0$ must then be treated separately.

REMARK 1. Condition (5) is used so that the linearization of $\left(1^{+}\right)$ and (1-) about the trivial solution $y \equiv 0$ is equation (7). 
REMARK 2. Examples of equations similar to $\left(1^{+}\right)$or $\left(1^{-}\right)$, although not necessarily of second order, can be found in the theory of "buckling" of elastic plates, shells, and rods. In the buckling of a straight rod or plate when the load parameters (in our case $\lambda$ ) is decreased the system usually returns to its unbuckled state (super-critical buckling). However in the buckling of shells and curved rods a "snap through" phenomenon may occur, where the deflections become large even though the load is decreased (sub-critical buckling). The behavior in the first case is associated with solutions of $\left(1^{-}\right)$and that of the second case with solutions of $\left(1^{+}\right)$.

REMARK 3. The physical significance of the nontrivial solutions mentioned in Theorem 2 for $\lambda \in(-\infty, 0)$ is usually associated with those kinds of "buckled" states which cannot be reached by a continuous deformation from the unbuckled state (trivial solution). In other words, these solutions do not bifurcate from the trivial solution $y \equiv 0$.

\section{REFERENCES}

1. M. Berger, A Sturm-Liouville theorem for non-linear elliptic partial differential equations, Ann. Scuola di Pisa 20 (1966), 543-582.

2. F. Browder, Variational methods for non-linear elliptic eigenvalue problems, Bull. Amer. Math. Soc. 71 (1965), 176-183.

3. N. Levinson, Positive eigenfunctions for $\Delta u+\lambda f(u)=0$, Arch. Rational Mech. Anal. 11 (1962), 258-272.

4. L. Ljusternik, Sur une class d'équations différentielles non-lineaires, Mat. Sb. 44 (1937), 1143-1168.

5. Z. Nehari, Characteristic values associated with a class of non-linear second order differential equations, Acta Math. 105 (1961), 141-175.

6. G. H. Pimbley, A sublinear Sturm-Liouville problem, J. Math. Mech. 11 (1962), 121-138.

7. — A super-linear Sturm-Liouville problem, Trans. Amer. Math. Soc. 103 (1962), 229-248.

8. J. H. Wolkowisky, Existence of buckled states of circular plates, Comm. Pure Appl. Math. (3) 20 (1967), 549-560.

Courant Institute of Mathematical Sciences, New York University 